\title{
Management of Lupus Nephritis
}

\section{Fernanda Payan Schober, MD Mary Anne Dooley, MD*}

\author{
Address \\ *Division of Nephrology and Hypertension, Department of Medicine, University of \\ North Carolina (UNC) Kidney Center, 7024 Burnett Womack Bldg. Campus Box \\ 7155, Chapel Hill, NC, 27599-7155, USA \\ Email: mary_dooley@med.unc.edu
}

Published online: 5 July 2016

(C) Springer International Publishing AG 2016

This article is part of the Topical Collection on Lupus

Keywords Lupus nephritis treatment · Systemic lupus erythematosus $\cdot$ Remission

\section{Opinion statement}

Systemic lupus erythematosus (SLE) is characterized by frequent renal involvement in approximately $50 \%$ of patients. Lupus nephritis (LN) remains the most important predictor of morbidity and mortality for patients with SLE. A recent international inception cohort reported $80 \%$ of patients develop LN within the first 2 years of disease; thus, it is imperative to screen SLE patients frequently early in disease and at routine clinical visits thereafter. Patients with proteinuria, active urinary sediment, or decreased effective glomerular filtration rate (eGFR) without other explanation should undergo a kidney biopsy to determine the histologic class and chronicity of LN to guide therapy. In general, we advocate initial treatment of proliferative lupus nephritis with intravenous (IV) cyclophosphamide according to the Euro-Lupus regimen. After initial treatment with cyclophosphamide, we transition patients to maintenance immunosuppression with mycophenolate mofetil or azathioprine for at least 3 to 5 years. Recent maintenance trials in LN report an increased proportion of patients achieving complete remission and fewer renal flares with this approach. Failure to attain a complete remission and renal flares are highly associated with progression to ESKD. Recently, repeat biopsy studies report substantial discordance between clinical measures of complete remission with up to $30 \%$ of patients showing continued histologic activity despite these features. We advocate that patients undergo repeat renal biopsy to ensure complete histological remission prior to discontinuing immunosuppressive therapy. In recent LN trials, only $50 \%$ of patients achieve complete or partial remission at 6 months. Patients with lupus nephritis should preferentially be enrolled in clinical trials as newer agents are needed to increase the proportion of patients responding to therapy. Patients also benefit greatly from more frequent monitoring and additional clinical support in trials. While recent clinical trials have not been successful including epratuzumab, tabalumab, and abatacept, promising new agents are in early phase trials. 


\section{Introduction}

Systemic lupus erythematosus (SLE) is a chronic, inflammatory, autoimmune disorder that can affect virtually any organ system. Kidney involvement is common with up to $50 \%$ of patients developing lupus nephritis and remains the single most important predictor of morbidity and mortality for patients with SLE [1]. The majority of patients with lupus nephritis (LN) (80\%) present within the first 2 years of their SLE diagnosis [2•]. Incidence of LN differs by ethnicity/race, with white patients less likely to have lupus nephritis (12$33 \%$ ) than black (40-69 \%), Hispanic (36-61\%) or Asian $(47-53 \%)$ patients $[1,3]$. Among patients with $\mathrm{LN}$, black patients have a worse prognosis with higher rates of progression to end-stage kidney disease (ESKD) compared to white patients with lupus nephritis [4-6].

The treatment approach to patients with LN involves characterizing the histopathologic class of with a kidney biopsy. According to the American College of Rheumatology (ACR) guidelines, the following SLE patients should have a kidney biopsy performed to evaluate for LN: [7]

1. Increasing serum creatinine without compelling alternative causes (such as sepsis, hypovolemia, or medication)
2. Confirmed proteinuria of $\geq 1 / 24$ h (24-h urine specimen or spot urine protein/creatinine ratios are acceptable)

3. Proteinuria $\geq 0.5 \mathrm{~g} / 24 \mathrm{~h}$ plus hematuria (defined as $\geq 5$ RBCs per hpf) or cellular casts There are six histopathologic classes of LN, which are described in Table 1 [7]. Treatment with immunosuppressive agents is typically confined to patients with active proliferative lesions (classes III and IV) or membranous LN with high risk features (nephrotic-range proteinuria or low eGFR). Treatment starts with an induction phase to induce remission, followed by maintenance therapy, with lesser toxicity. This terminology implies that remission is achieved by the induction phase, although this is frequently not the case $[3,8]$. In fact, recent clinical trials using mycophenolate mofetil, rituximab, abatacept, and cyclophosphamide have shown that only $50 \%$ of patients achieve either complete or partial remission at 6 months of therapy $[8,9 \bullet, 10 \bullet]$.

\section{Treatment options $\rightarrow$}

\section{Pharmacologic treatment}

\section{Initial therapy for lupus nephritis}

Historically, therapy for LN consisted solely of corticosteroid therapy. Beginning in the 1970s, several trials evaluated the efficacy of adding immunosuppressive agents to steroid regimens. Early studies using azathioprine or cyclophosphamide in addition to steroids were inconclusive [11-17]. A meta-analysis of these studies reported that patients with LN had better preserved renal function, less progression to ESKD, and fewer deaths from kidney disease in the arms including immunosuppressive drugs with steroids rather than steroids alone [18]. The pivotal NIH randomized trial compared prednisone alone to treatment with azathioprine, oral cyclophosphamide, or intravenous (IV) cyclophosphamide, reporting that immunosuppressive therapy dramatically decreased the rate of kidney failure, establishing immunosuppressive therapy, particularly intravenous cyclophosphamide therapy as the mainstay for LN [19]. 


\section{Table 1. ISN/RPS lupus nephritis 2004 classification}

\begin{tabular}{ll}
\hline Class I & Mesangial immune deposits without mesangial hypercellularity \\
Class II & Mesangial immune deposits with mesangial hypercellularity \\
Class III & Focal (involving $<50 \%$ of total glomeruli) glomerulonephritis \\
III (A) & Active lesions \\
III (C) & Chronic lesions \\
Class IV & Diffuse (involving $\geq 50 \%$ of total glomeruli) glomerulonephritis \\
IV-S & Segmental lesions ( $<50 \%$ of the glomerular tuft is affected) \\
IV-G & Global lesions ( $\geq 50 \%$ of the glomerular tuft is affected) \\
Class V & Membranous lupus nephritis \\
Class VI & Advanced sclerosing lesions $(>90 \%$ of total glomeruli)
\end{tabular}

Prednisone remains the backbone of induction therapy with IV methylprednisolone typically used at the beginning of treatment. Many studies have used $1 \mathrm{~g}$ daily for 3 days, but given the side effects and general toxicity of high-dose steroid use, we recommend using $500 \mathrm{mg}$ daily for 3 days or $7 \mathrm{mg} / \mathrm{kg}$ (whichever is lower). After the initial use of IV methylprednisolone, oral prednisone should be initiated at a dose of $1 \mathrm{mg} / \mathrm{kg}$ with a maximum daily dose of $60 \mathrm{mg}$. There are no established guidelines for tapering prednisone, but the general consensus is that corticosteroids use should be shortened in SLE. We propose the following prednisone taper schedule (Table 2). Extra-renal lupus manifestations often hinder the ability to taper prednisone completely but the goal should be $\leq 100 \mathrm{mg}$ daily to avoid the toxic side effects of chronic glucocorticoid steroid use. Prior to starting prednisone, patients should be warned about the potential side effects including weight gain, edema, increased blood pressure, elevated blood sugars, osteoporosis, mood swings, insomnia, and increased risk of gastrointestinal bleeding. Patients on long-term prednisone should be prescribed an acidreducing agent to prevent gastritis and calcium with vitamin $\mathrm{D}$ to promote bone health. Patients with diabetes mellitus should be monitored frequently as they may need adjustments to their diabetes medications.

\section{Induction therapy for lupus nephritis}

Cyclophosphamide is an alkylating agent with anti-cancer and immunosuppression properties. By adding an alkyl group to DNA, it interferes with DNA replication making rapidly dividing cells particularly sensitive to the effects of this medication. Side effects of this medication include bone 


\title{
Table 2. Proposed prednisone taper
}

\author{
Prednisone taper \\ Prednisone $60 \mathrm{mg}$ P0 daily $\times 14$ days \\ Prednisone $40 \mathrm{mg}$ P0 daily $\times 14$ days \\ Prednisone $35 \mathrm{mg}$ PO daily $\times 7$ days \\ Prednisone $30 \mathrm{mg} \mathrm{PO}$ daily $\times 7$ days \\ Prednisone $25 \mathrm{mg}$ P0 daily $\times 7$ days \\ Prednisone $20 \mathrm{mg} P 0$ daily $\times 7$ days \\ Prednisone $15 \mathrm{mg}$ P0 daily $\times 7$ days \\ Prednisone $10 \mathrm{mg}$ P0 daily $\times 7$ days by week 12 \\ Prednisone $7.5 \mathrm{mg} P 0$ daily $\times 7$ days \\ Prednisone $5 \mathrm{mg}$ PO daily $\times 7$ days \\ Prednisone $2.5 \mathrm{mg}$ PO daily $\times 7$ days then OFF \\ It is $\mathrm{OK}$ to modify this prednisone taper as needed during disease course. Some patients may not be able to stop prednisone completely, but \\ prednisone $10 \mathrm{mg}$ daily by week 12 is strongly encouraged
}

marrow suppression, increased risk of infection, hemorrhagic cystitis, bladder cancer, and gonadal failure. The risk of gonadal failure is of particular concern given that the treatment population is predominately women of childbearing age.

In several randomized controlled trials, the addition of cyclophosphamide decreased the risk of progression to end-stage kidney failure [20-22]. A "short course" of cyclophosphamide (six monthly doses of IV cyclophosphamide dosed $0.5 \mathrm{~g}-1 \mathrm{~g} / \mathrm{m}^{2)}$ was compared to a "long-course" cyclophosphamide (six monthly doses of IV cyclophosphamide $\left(0.5 \mathrm{~g}-1 \mathrm{~g} / \mathrm{m}^{2}\right)$, followed by quarterly doses for 2 years. Patients receiving long-course cyclophosphamide were less likely to flare their disease and were more likely to have preserved kidney function [20]. Long-course cyclophosphamide, known as the "NIH protocol," became the standard of care for LN induction therapy for years.

Subsequent studies evaluated reduced dosing of cyclophosphamide in an effort to decrease toxic side effects while maintaining efficacy. The EuroLupus trial randomized patients to NIH protocol IV cyclophosphamide over 2 years or to a lower dose of cyclophosphamide, which was $500 \mathrm{mg}$ IV every 2 weeks for six total doses. After 24 weeks of follow-up, there was no statistical difference in remission between these two groups with numerically fewer episodes of severe infection in the low-dose group, although the difference was not statistically significant. Patients enrolled in the EuroLupus trial were predominately white Europeans ( $84 \%)$ and few patients had severe kidney disease [22]. Ten-year follow-up of these patients has shown no difference in LN relapses, death, sustained doubling of serum creatinine, or ESKD between the two groups [23].

The Abatacept and Cyclophosphamide Combination Efficacy and Safety Study (ACCESS) trial treated 134 patients with cyclophosphamide according to the Euro-Lupus regimen and randomized patients to additional treatment with abatacept or placebo. Over $30 \%$ of patients in both groups 
had a complete response and $59 \%$ had a partial response at 24 weeks of treatment with Euro-Lupus cyclophosphamide; though there was no difference between abatacept and placebo, patients enrolled in this study were from the USA and Mexico, so they were more ethnically and racially diverse than patients in the original European study, increasing the applicability of low-dose cyclophosphamide to non-white patients [10•].

Given the toxic effects of cyclophosphamide, we propose that Euro-Lupus cyclophosphamide be used as initial therapy for LN. While it may be inconvenient for patients to have infusion appointments every 2 weeks, the additional benefit of this frequent cyclophosphamide dosing schedule is that it allows healthcare providers to have frequent contact with patients during this crucial and tenuous time.

The exception to this recommendation is in patients with certain pathologic characteristics that are associated with more aggressive kidney disease. Tuft necrosis is an uncommon histopathologic finding seen in proliferative lupus nephritis LN that is associated with rapid loss of kidney function and progression to ESKD [6]. It resembles anti-neutrophil cytoplasmic antibody (ANCA) glomerulonephritis in that the lesions are more focal and segmental with fibrinoid necrosis, which may explain the poor prognosis in these patients $[6,24]$. We propose that these individuals be treated with 6 months of NIH cyclophosphamide, which is similar to an ANCA glomerulonephritis treatment regimen. Thorough review with the nephropathologist evaluating the kidney biopsy is needed to determine the extent of kidney activity/severity which will determine the optimal cyclophosphamide dosing regimen. Of note, patients receiving NIH cyclophosphamide will start with $0.5 \mathrm{~g} / \mathrm{m}^{2}$. A complete blood cell count needs to be checked 2 weeks after each monthly dose as this is when the white blood cells reach their nadir. If patients are not neutropenic, then the cyclophosphamide dose should be increased each subsequent month to a maximum dose of $1 \mathrm{~g} / \mathrm{m}^{2}$.

Oral cyclophosphamide $1.0-1.5 \mathrm{mg} / \mathrm{kg} /$ day (maximum dose $150 \mathrm{mg} /$ day) for 2-4 months has been used as an alternative to IV cyclophosphamide as it is less expensive and may be easier to administer in areas that lack access to an infusion center. However, it is not clear whether it has equivalent efficacy to IV cyclophosphamide and more adverse effects have been reported with oral therapy compared to IV cyclophosphamide, so this is not recommended unless IV is not a feasible option $[8,22]$.

Cyclophosphamide is contraindicated in pregnancy. Female patients of childbearing age should have a pregnancy test performed prior to each dose. The role of gonadotropin-releasing hormone analogs (GnRHa) to prevent gonadal toxicity will be discussed in a later section.

Azathioprine is a purine antimetabolite used as an immunosuppressant for prevention of organ transplant rejection and treatment of autoimmune disorders. The Dutch Lupus Nephritis Study randomized patients with proliferative LN to either NIH dosing IV cyclophosphamide for 2 years or therapy with daily azathioprine and steroids and found no difference in kidney response during the first 2 years of treatment [25]. However, 
subsequent 10-year follow-up showed that the azathioprine group had a higher rate of relapse and more chronicity on subsequent kidney biopsies, although there was no significant difference in sustained doubling of serum creatinine [26•]. Even so, we do not recommend azathioprine as initial therapy to induce remission in patients with $\mathrm{LN}$.

Mycophenolate mofetil

Calcineurin inhibitors

Mycophenolate mofetil, an immunosuppressive drug approved for prevention of transplant rejection, has been proposed as an alternative to initial therapy with cyclophosphamide. A randomized, non-inferiority trial showed that mycophenolate mofetil was more effective at inducing remission than six monthly doses of IV cyclophosphamide at 24 weeks [27]. This was followed by a large international randomized control trial, the Aspreva Lupus Management Study (ALMS), which randomized 370 patients to either 24 weeks of induction therapy with monthly IV cyclophosphamide or daily oral mycophenolate mofetil (1000 mg BID initially and increased to $1500 \mathrm{mg}$ BID if tolerated) and found no significant difference in response rates, which the study defined as a decrease in urine protein to creatinine ratio and stabilization or improvement in serum creatinine, between the two groups. Secondary sub-analysis revealed that fewer Hispanic and black patients responded to cyclophosphamide compared to mycophenolate mofetil [11].

A drawback of initial therapy with mycophenolate mofetil is that patients have to take the medication twice a day, so there is the potential for nonadherence. With IV cyclophosphamide treatments, the ordering providers can be certain that patients have received the medication.

This is an expensive medication, particularly if patients do not have health insurance. Patients may experience gastrointestinal symptoms when starting this medication, so we typically advise patients to start with $500 \mathrm{mg}$ twice a day (BID) and increase the dose to $1000 \mathrm{mg}$ BID after 5-7 days. If patients can tolerate this dose, then it can be increased further to $1500 \mathrm{mg}$ BID, which is the maximum dose. Mycophenolate mofetil can cause leukopenia, anemia, and thrombocytopenia, so patients should have their blood counts checked while on the medication and prior to increasing dose from 2 to $3 \mathrm{~g}$ daily. This medication is also teratogenic, so women of childbearing age need to be counseled on the importance of pregnancy prevention and should be on a reliable form of birth control.

Calcineurin inhibitors have been investigated as well for remission induction in LN, but have yielded disappointing results.

The Cyclofa-Lune study randomly assigned 40 patients with LN to induction therapy with cyclosporine or cyclophosphamide. Patients treated with cyclosporine received $4-5 \mathrm{mg} / \mathrm{kg} / \mathrm{day}$ for 9 months, and then the dose was gradually decreased for another 9 months. Cyclophosphamide was given in eight IV boluses over 9 months, followed by an additional 9 months of oral cyclophosphamide therapy. There was no significant difference in relapsefree survival between the two groups after 40 months of follow-up [28]. 
Small, randomized controlled trials have compared 24 weeks of initial treatment with tacrolimus or monthly IV cyclophosphamide and have not seen any differences in kidney response. However, these studies have been of short duration and do not have data on long-term renal response after discontinuation of treatment $[29,30]$. Additionally, follow-up kidney biopsies have shown more chronicity and scarring [31]. We do not recommend initial treatment with a calcineurin inhibitor alone for proliferative LN.

The Lupus Nephritis Assessment with Rituximab Trial (LUNAR) evaluated the efficacy and safety of rituximab, an anti-CD20 monoclonal antibody, in a randomized trial. Patients were randomized to receive rituximab versus placebo in addition to standard therapy with mycophenolate mofetil and prednisone. While rituximab led to a greater serologic response in dsDNA and complement levels, clinical outcomes were unchanged at 1 year [ $9 \bullet]$.

\section{Maintenance therapy for lupus nephritis}

After initial therapy, patients must continue daily immunosuppressive therapy to maintain remission. In most cases, we recommend the use of mycophenolate mofetil for maintenance therapy as this has been shown to be superior to azathioprine in maintaining a kidney response and preventing relapse [32]. The exceptions to this recommendation are in women patients that who cannot tolerate the side effects of mycophenolate mofetil or in women that who are pregnant or contemplating pregnancy as mycophenolate mofetil is teratogenic and must be avoided during pregnancy.

Patients that who participated in the Aspreva Lupus Management Study (ALMS) trial previously mentioned received initial treatment for 24 weeks with IV cyclophosphamide or mycophenolate mofetil and were subsequently randomized to maintenance therapy with either mycophenolate mofetil or azathioprine for an additional 36 months. Those patients that who received mycophenolate maintenance therapy were less likely to relapse than those patients that who received azathioprine maintenance therapy regardless of race or choice of initial therapy [10•].

This finding was corroborated by the MAINTAIN trial which randomized patients to maintenance therapy with either mycophenolate mofetil or azathioprine after initial therapy for 9 months with IV cyclophosphamide (given as $500 \mathrm{mg}$ every 2 weeks according to the Euro-Lupus regimen). Patients were treated for a minimum of 3 years, with a mean follow-up of 48 months. While not statistically significant, patients treated with mycophenolate mofetil were less likely to have a renal relapse. Interestingly, this study also included protocol biopsies at 2 years and did not find a significant histologic difference between the two groups [25,33].

A meta-analysis comparing mycophenolate mofetil versus azathioprine as maintenance therapy for LN found no significant difference between the two medications in terms of relapse prevention, progression to ESKD, doubling of serum creatinine or death. However, mycophenolate mofetil was generally better tolerated with fever patients developing leukopenia or amenorrhea [34].

Duration of maintenance therapy is unclear, but most studies have 
maintained patients on therapy with either mycophenolate mofetil or azathioprine for at least 30 months $[11,25,35]$. Given the high risk of relapse, we recommend that patients remain on extended maintenance therapy for at least 3 years. Prior to cessation of maintenance therapy, one should consider performing a repeat kidney biopsy to ensure that there is no active kidney involvement. Studies have shown that despite apparent clinical quiescence and normal urine sediment evaluations, a high percentage of patients that are thought to be in complete remission can have active nephritis $[35,36]$.

\section{Treatment of non-proliferative lupus nephritis}

Class V LN, also referred to as lupus membranous, affects $10-20 \%$ of patients with LN. It is discussed here separately since all of the trials previously mentioned included patients with proliferative lesions. Only one randomized clinical trial has focused solely on patients with pure membranous lesions without endocapillary proliferation. Forty-two patients were randomized to treatment with prednisone alone, cyclosporine for 11 months or monthly infusions of IV cyclophosphamide for 6 months. While patients treated with cyclosporine were more likely to be in remission than patients treated with prednisone alone or cyclophosphamide, extended follow-up showed that these patients were far more likely to relapse than the patients treated with cyclophosphamide [37].

A pooled analysis of the ALMS and Ginzler trials that used using cyclophosphamide versus mycophenolate mofetil as initial therapy identified 84 patients with pure class V LN and found that there was no difference in serum creatinine or proteinuria at 24 weeks in this subset of patients [38].

There are no treatment guidelines for isolated lupus membranous, but the general consensus is that patients with nephrotic-range proteinuria should be treated with immunosuppression in additional to an ace-inhibitor or angiotensin receptor blocker. However, there is no consensus as to which immunomodulating agent to use and whether patients without nephroticrange proteinuria should be treated [3]. We recommend treating these patients with mycophenolate mofetil initially and then adding a calcineurin inhibitor if they continue to have over $1 \mathrm{~g}$ of daily proteinuria.

"Point of no return"

Patients with class VI LN, characterized as $>90 \%$ sclerosis, are unlikely to improve with use of immunosuppressive therapy. These patients should not be exposed to cytotoxic agents and all of the negative side effects of these medications. They should be referred for kidney transplant and should receive hemodialysis and or peritoneal dialysis education and planning.

\section{Other considerations}

1. Cyclophosphamide and mycophenolate mofetil are teratogenic, so patients must be counseled regarding the contraindication for pregnancy and be on a reliable form of contraception prior to initiating either medication.

2. There is a risk of premature ovarian failure associated with use of cyclophosphamide. The risk is highest in males,including pre-pubertal boys, and 
no preventive strategy has been developed. In females, the risk increases after puberty with patient's age and with higher cyclophosphamide doses [39]. The use of gonadotropin-releasing hormone analogs (GnRHa) to protect against premature ovarian failure from cyclophosphamide was studied in a population of patients with $\mathrm{LN}$ receiving NIH dosing monthly cyclophosphamide. Depot leuprolide acetate was administered once a month, 2 weeks prior to the monthly IV cyclophosphamide dose. The treated group had a significant reduction in premature ovarian failure ( 5 versus $30 \%$ ) [40]. It seems reasonable to provide women of childbearing age the option of treatment with a gonadotropin-releasing hormone analog while receiving cyclophosphamide therapy. However, cyclophosphamide treatment should never be delayed while awaiting GnRHa therapy. The risks of therapy include increased risk of blood clotting in a population who may be nephrotic and/or antiphospholipid antibody positive, osteoporosis, menopausal symptoms and altered lipid profile. Another benefit of treatment with the Euro-Lupus treatment protocol is that patients receive a lower cumulative dose of IV cyclophosphamide, lowering the risk of gonadal toxicity. Use of a 3 month depot GnRHa may be used as well, which is given once prior to therapy (ideally 2 weeks before) and lasts for 3 months.

3. Patients receiving immunosuppression should not receive any live vaccines. These include measles, mumps, rubella, influenza nasal spray, varicella, oral polio vaccines, rotavirus, and yellow fever [41, 42].

\section{Pregnancy}

Pregnancy should be delayed until complete remission has been achieved. Data suggest that pregnancy during active LN results in a significantly higher risk of fetal loss [43]. Pregnancy during active lupus nephritis also results in worsening kidney function, which is why it is important that patients be counseled regarding pregnancy prevention until they are in remission.

Patients with LN who are being treated with mycophenolate mofetil should be switched to azathioprine prior to pregnancy. If patients remain in remission for at least 3 months on azathioprine, then pregnancy can be pursued [3]. Patients should be monitored during pregnancy by a high-risk obstetrician. Additionally, women who have antibodies to Ro/SSA and/or La/SSB should undergo fetal monitoring for heart block as this affects $2 \%$ of primigravid mothers with these antibodies [44]. Patients with SLE and anticardiolipin antibodies should receive low-dose aspirin therapy during pregnancy [45]. Hydroxychloroquine should be continued during pregnancy to improve outcomes.

\section{Emerging therapies}

\section{Belimumab}

Patients with SLE have higher levels of soluble B-lymphocyte stimulator (BLyS) which is a key survival cytokine for B lymphocytes. Belimumab is a human immunoglobulin monoclonal antibody that inhibits the biologic activity of BLyS. There have been two phase 3 trials, BLISS-52 and BLISS-76, 
which tested the addition of belimumab to standard SLE therapy. These two trials were not designed to specifically assess the effects of belimumab on renal parameters as patients with severe active LN were excluded [46, 47]. However, post hoc analysis of these studies has revealed that patients with lupus kidney involvement had an improvement if treated with belimumab compared to placebo [48•].

There is an ongoing trial, BLISS-LN, which is a phase 3, randomized, double blind, placebo-controlled study, which randomizes patients with active LN to belimumab or placebo in addition to standard induction therapy with either mycophenolate mofetil or cyclophosphamide (NCT01639339).

Voclosporin is a new calcineurin inhibitor that has been engineered to have a safer side-effect profile than cyclosporine. The efficacy of this medication is currently being tested in patients with $\mathrm{LN}$, including patients that have isolated lupus membranous without proliferative lesions (NCT02141672). This is the only current clinical trial that is enrolling patients with pure class V LN.

Abatacept is a human, soluble fusion protein that blocks the binding of antigen-presenting cells to CD28 on T cells, therefore inhibiting activation of primary $\mathrm{T}$ cell-dependent immune responses. In murine models, it has been shown to work synergistically with cyclophosphamide to arrest LN. Unfortunately, as mentioned earlier, it has not been shown to have this additional benefit in humans as the ACCESS trial failed to show an increase rate of remission in the treatment arm compared to placebo [13].

TWEAK is a soluble cytokine expressed primarily by leukocytes which is upregulated locally on epithelial and mesenchymal cells in injured and diseased tissues. It binds to its receptor, Fn14, to mediate pro-inflammatory responses including vascular activation, angiogenesis, cell growth, and cell death. Urinary TWEAK levels have been found to be elevated in patients with LN compared to patients with lupus and no renal disease. AntiTWEAK is a monoclonal antibody that inhibits TWEAK binding to Fn14. There is a clinical trial currently being conducted to assess the efficacy of anti-TWEAK in addition to background LN therapy compared with placebo (NCT01499355).

\section{Recent therapeutic failures}

The following is a list of biologic therapies that have recently been deemed unsuccessful for treatment of SLE, but have not been tested in patients with LN specifically.

Tabalumab is an anti-B cell activating factor (BAFF) human IgG4 monoclonal antibody. Patients with SLE, excluding patients with LN or cerebritis, 
were enrolled in two phase 3, placebo controlled, clinical trials called ILLUMINATE I and II. The trials were terminated early as the tabalumab group did not meet primary end-points [49].

Epratuzumab is a monoclonal antibody that targets CD22, resulting in decreased B cell activity without depleting B cells. The two phase 3 trials called EMBODY failed to meet their primary clinical efficacy endpoints with either dose [50].

Sirukumab is an interleukin-6 antagonist that was being investigated in a randomized, placebo-controlled trial. Unfortunately, the trial was stopped due to a high rate of adverse events, particularly infections [51].

\section{Promising future agents}

Sifalimumab is an anti-interferon-alpha monoclonal antibody that is currently being investigated in treatment of lupus. Phase 2 clinical studies showed reduced SLE activity and had an acceptable safety profile, so phase 3 studies will need to be completed (NCT01283139) [52].

Anifrolumab is a monoclonal antibody against type 1 interferon (IFN) that has recently been shown to improve symptoms of lupus such as rash and arthritis. Patients are currently being recruited for phase 3 studies (NCT02446899) [53].

\section{Conclusions}

Despite advances in treatment regimens for $\mathrm{LN}$, it remains the cause of significant morbidity and mortality for patients with SLE. Since half of patients with SLE will have kidney involvement, it is imperative that clinicians caring for patients with SLE screen for LN. If there is a clinical suspicion for LN, a kidney biopsy must should be performed to classify the histopathologic lesions. Patients with proliferative LN (class III/IV) should be treated with IV cyclophosphamide for 3 months, followed by daily mycophenolate mofetil. Prednisone should be weaned off as soon as possible, particularly in patients with LN who have no extra-renal manifestations. There are a number of novel therapies being explored for treatment of SLE, which will hopefully also have a role in treatment of LN. There has only been one new medication approved for treatment of SLE in the last 50 years, so there is a great need for new therapeutic agents to improve the kidney and overall patient survival in patients with SLE.

\section{Compliance with Ethical Standards}

\section{Conflicts of Interest}

Fernanda Payan Schober declares that she has no conflict of interest. Mary Anne Dooley declares that she has no conflict of interest. 
Human and Animal Rights and Informed Consent

This article does not contain any studies with human or animal subjects performed by any of the authors.

\section{References and Recommended Reading}

Papers of particular interest, published recently, have been

highlighted as:

- Of importance

1. Adler M, Chambers S, Edwards C, Neild G, Isenberg D. An assessment of renal failure in an SLE cohort with special reference to ethnicity, over a 25 -year period. Rheumatology. 2006;45:1144-7.

2. Hanly J. et al. The frequency and outcome of lupus nephritis: results from an international inception cohort study. Rheumatology. 2015.

Of the 1827 patients in the Systemic Lupus International Collaborating Clinics (SLICC) inception cohort that were assessed annually (mean 4.6 years), 38.3\% developed LN. Patients with $\mathrm{LN}$ were younger, more likely to be men and were black, Asian or Hispanic. LN was associated with ESKD and death.

3. Kidney Disease: Improving Global Outcomes (KDIGO) Glomerulonephritis Work Group. KDIGO Clinical Practice Guidelines for Glomerulonephritis. Kidney Inter Suppl. 2012;2:139-274.

4. Ward MM. Changes in the incidence of end-stage renal disease due to lupus nephritis, 1982-1995. Arch Intern Med. 2000;160:3136-40.

5. Ward MM. Changes in the incidence of end-stage renal disease due to lupus nephritis in the United States, 1996-2004. J Rheumatol. 2009;36(1):63-7.

6. Korbet SM, Schwartz M, Evans J, Lewis E. Severe lupus nephritis: racial differences in presentation and outcome. J Am Soc Nephol. 2007;18(1):244-54.

7. Weening J et al. The classification of glomerulonephritis in systemic lupus erythematosus revisited. JASN. 2004; 15:241-25.

8. Appel G, The Aspreva Lupus Management Study Group. Mycophenolate mofetil versus cyclophosphamide for induction treatment of lupus nephritis. J Am Soc Nephrol. 2009;20:1103-12.

9. Rovin B, Furie R, Latinis $\mathrm{K}$, et al. Efficacy and safety of rituximab in patients with active proliferative lupus nephritis. Arthritis Rheum. 2012;64(4):1215-26.

Randomized, phase III trial to evaluate the efficacy and safety of rituximab in addition to cyclophosphamide in treatment of LN. Despite appropriate peripheral B cell depletion in the rituximab group, there was no statistical improvement in kidney response rates with addition of rituximab.

10. The ACCESS trial group. Treatment of lupus nephritis with abatacept: the abatacept and cyclophosphamide combination efficacy and safety study. Arthritis Rheum. 2014;66:3096-104.

Randomized, phase II trial of abatacept versus placebo in addition to cyclophophosphamide in patients with active LN. There were 134 patients enrolled and there was no statistically significant difference in compete response at week 24 .

11. Donadio Jr JV, Holley KE, Wagoner RD, Ferguson RH, McDuffie FC. Treatment of lupus nephritis with prednisone and combined prednisone and azathioprine. Ann Intern Med. 1972;77:829-35.

12. Donadio Jr JV, Holley VE, Ferguson RH, Illstrup DM. Progressive lupus glomerulonephritis: treatment with prednisone and combined prednisone and cyclophosphamide. Mayo Clin Proc. 1976;51:484-94.

13. Sztenjnbock M, Steart A, Diamond H, et al. Azathioprine in the treatment of systemic lupus erythematosus. A controlled study. Arthritis Rheum. 1971;14:639-45.

14. Cade R, Spooner G, SChlein E, et al. Comparison of azathioprine, prednisone, and heparin alone or combined in treating lupus nephritis. Nephron. 1973;10:37-56.

15. Steinberg AD, Decker JL. A double-blind controlled trial comparing cyclophosphamide, azathioprine and placebo in the treatment of lupus glomerulonephritis. Arthritis Rheum. 1974;17:923-37.

16. Steinberg AD, Kaltreider HB, Staples PJ, Goetzl EJ, Talal $\mathrm{N}$, Decker JL. Cyclophosphamide in lupus nephritis: a controlled trial. Ann Intern Med. 1971;75:165-71.

17. Dinant HJ, Decker JL, Klippel JH, et al. Alternative modes of cyclophosphamide and azathioprine therapy in lupus nephritis. Ann Intern Med. 1983;96(6):728-36.

18. Felson DT, Anderson J. Evidence for the superiority of immunosuppressive drugs and prednisone over prednisone alone in lupus nephritis. N Engl J Med. 1984;311(24):1528-33.

19. Austin $\mathrm{H}$ et al. Therapy of lupus nephritis: controlled trial of prednisone and cytotoxic drugs. N Engl J Med. 1986;314:614-9.

20. Boumpas D. Controlled trial of pulse methylprednisolone versus two regimens of pulse cyclophosphamide in severe lupus nephritis. Lancet. 1992;340:741-5.

21. Gourley MF et al. Methylprednisolone and cyclophosphamide, alone or in combination, in patients with lupus nephritis: a randomized, controlled trial. Ann Intern Med. 1996;125(7):549-57.

22. Houssiua $\mathrm{F}$ et al. Immunosuppressive therapy in lupus nephritis: the Euro-Lupus Nephritis Trial, a randomized trial of low-dose versus high-dose intravenous cyclophosphamide. Arthritis Rheum. 2002;46(8):2121-31.

23. Houssiau F et al. The 10-year follow-up data of the Euro-Lupus Nephritis Trial comparing low-dose and high-dose intravenous cyclophosphamide. Ann Rheum Dis. 2010;69:2083-9. 
24. Hill G et al. Class IV-S versus class IV-G lupus nephritis: clinical and morphologic differences suggesting different pathogenesis. Kidney Int. 2005;68:2288-97.

25. Grootscholten C et al. Azathioprine/ methylprednisolone versus cyclophosphamide in proliferative lupus nephritis. A randomized controlled trial. Kidney Int. 2006;70:732-42.

26. Arends S, on behalf of the Dutch Working Party, et al. Long-term follow-up of a randomized controlled trial of azathioprine/methylprednisolone versus cyclophosphamide in patients with proliferative lupus nephritis. Ann Rheum Dis. 2012;71:966-73.

This study reported 10-year follow-up of the original Dutch Working Party study which randomized 87 patients with LN to induction therapy with either azathioprine or IV cyclophosphamide. There were more relapses in the azathioprine group, but sustained doubling of serum creatinine, ESKD and mortality did not differ between the two groups.

27. Ginzler E et al. Mycophenolate mofetil or intravenous cyclophosphamide for lupus nephritis. N Engl J Med. 2005;353(21):2219-28.

28. Zavada J et al. Cyclosporine A or intravenous cyclophosphamide for lupus nephritis: the Cyclofa-Lune study. Lupus. 2010;19:1281-9.

29. Chen W et al. Short-term outcomes of induction therapy with tacrolimus versus cyclophosphamide for active lupus nephritis: a multicenter randomized clinical trial. AJKD. 2011;57(2):235-44.

30. Li Z et al. Mycophenolate mofetil or tacrolimus compared with intravenous cyclophosphamide in the induction treatment for active lupus nephritis. Nephrol Dial Transplant. 2012;4:1467-77.

31. Radhakrishna J, Kunis CL, D'Agati V, Appel GB. Cyclosporin treatment of lupus membraneous nephropathy. Clin Nephrol. 1994;42:147-54.

32. Dooley MA, Jayne D, Ginzler E, et al. Mycophenolate versus azathioprine as maintenance therapy for lupus nephritis. NEJM. 2011;365(2):1886-95.

33. Houssiau FA, D'Cruz D, Sangle S, et al. Azathioprine versus mycophenolate mofetil for long-term immunosuppression in lupus nephritis: results from the MAINTAIN Nephritis Trial. Ann Rheum Dis. 2010;69:2083-9.

34. Feng L. Mycophenolate mofetil versus azathioprine as maintenance therapy for lupus nephritis: a meta-analysis. Nephrology. 2013;18:104-10.

35. Zickert A, Sunderlin B, Svenungsson E, Gunnarsson I. Role of early repeated renal biopsies in lupus nephritis. Lupus Sci Med. 2014;1(1), e000018. doi:10.1136/ lupus-2014-000018.

36. Schober FP, Pendergraft WF. Dialogue: a blissful future for lupus nephritis: harnessing repeat kidney biopsies to identify meaningful biomarkers of disease. Lupus Sci Med. 2015;2(1), e000083. doi:10.1136/lupus-2015000083.

37. Austin $\mathrm{H}$ et al. Randomized, controlled trial of prednisone, cyclophosphamide, and cyclosporine in lupus membranous nephropathy. JASN. 2009;20(4):901-11.
38. Radhakrishnan J et al. Mycophenolate mofetil and intravenous cyclophosphamide are similar as induction therapy for class V lupus nephritis. Kidney Int. 2010;77:152-60.

39. Wang C, Wang F, Bosco JJ. Ovarian failure in oral cyclophosphamide treatment for systemic lupus erythematosus. Lupus. 1995;4:11-4.

40. Somers E et al. Use of a gonadotropin-releasing hormone analog for protection against ovarian failure during cyclophosphamide therapy in women with severe lupus. Arthritis Rheum. 2005;52(9):2761-7.

41. Patel RR et al. Travel advice for the immunocompromised traveler: prophylaxis, vaccination, and other preventive measures. Ther Clin Risk Manag. 2015;11:217-28.

42. Rubin, L. et al. 2013 IDSA Clinical practice guideline for vaccination of the immunocompromised host. Clin Infect Dis. 2013.

43. Wagner SJ, Craici I, Reed D, et al. Maternal and fetal outcomes in pregnant patients with active lupus nephritis. Lupus. 2009;18:342-7.

44. Buyon J, Clancy R, Friedman D. Cardiac manifestations of neonatal lupus erythematosus: guidelines to management, integrating clues from the bench and bedside. Nat Clin Pract Rheumatol. 2009;5:139-48.

45. Lateef A, Petri M. Managing lupus patients during pregnancy. Best Pract Res Clin Rheumatol. 2013;27(3):435-47. doi:10.1016/j.berh.2013.07.005.

46. Navarra SV, Guzman RM, Gallacher AE, BLISS-52 Study Group, et al. Efficacy and safety of belimumab in patients with active systemic lupus erythematosus. Lancet. 2011;377:721-31.

47. Furie R, Petri M, Zamari O, for the BLISS-76 Study Group, et al. A phase III, randomized, placebocontrolled study of belimumab, a monoclonal antibody that inhibits B lymphocyte stimulator, in patients with systemic lupus erythematosus. Arthritis Rheum. 2011;63:3918-30.

48. Dooley MA et al. Effect of belimumab treatment on renal outcomes: results from the phase 3 belimumab clinical trials in patients with SLE. Lupus. 2014;22:63-72.

Post-hoc analysis of both BLISS-52 and BLISS-76, which were two phase 3 trials evaluating the benefit of belimumab versus placebo to mycophenolate treatment for patients with SLE. There were 267 patients that had kidney involvement (patients with severe active LN were excluded from the trial) and post hoc analysis of these patients showed benefit of belimumab in terms of inducing renal remission.

49. Isenberg D et al. PO0184 Efficacy and safety of tabalumab in patients with systemic lupus erythematosus (SLE): results from 2 phase 3, 52week, multicenter, randomized, placebo-controlled trials. Ann Rheum Dis. 2015;74:141.

50. UCB announces phase 3 clinical trial program for epratuzumab in systemic lupus erythematosus did not meet primary endpoint. July 28, 2015, wwww.ucb.com/presenter/News/article. Accessed Dec 1, 2015. 
51. Sullivan, M. Trial of sirukumab for lupus nephritis falls flat. Rheumatol News Digit Netw. 2014.

52. MedImmune presents promising new data in systemic lupus at American College of Rheumatology Meeting. Business Wire. Nov 17, 2014. http://www. businesswire.com/news/home/20141117005295/en/ MedImmune-Presents-Promising-Data-SystemicLupus-American. Accessed 1 Dec 2015.
53. Anifrolumab met primary and secondary endpoints in phase II, significantly reducing lupus disease activity compared with placebo across multiple endpoints. November 10,2015. https://www.astrazeneca.com/ our-company/media-centre/press-releases/2015/

AstraZeneca-presents-positive-new-data-onanifrolumab-in-lupus-at-American-College-ofRheumatology-Annual-Scientific-Meeting-10112015. html. Accessed 1 Dec 2015. 